\title{
Consolidation Algorithm Interoperability in Point-to-Multipoint ABR Services in ATM Networks
}

\author{
Naris Rangsinoppamas ${ }^{1}$, Tanun Jaruvitayakovit ${ }^{1}$, Wisitsak Sa-niamsak ${ }^{2}$, \\ Praphan Pavarangkoon ${ }^{2}$, and Prasit Prapinmongkolkarn ${ }^{1}$ \\ 1 Telecommunication System Research Laboratory \\ Department of Electrical Engineering, Faculty of Engineering \\ Chulalongkorn University, Bangkok, Thailand \\ nrr@kmitnb.ac.th \\ 2 Telecommunication System Research Laboratory \\ Department of Electrical Engineering, Faculty of Engineering \\ King Mongkut Institute of Technology North Bangkok, Bangkok, Thailand
}

\begin{abstract}
The point-to-multipoint ABR service is a very important service in ATM networks. Recently, several researchers have studied the issues on multicast ABR services and have proposed a family of consolidation algorithms. All of the previous proposed algorithms have some drawbacks such as slow response, high consolidation noise and high implementation complexity etc. Hence, we propose a new efficient consolidation algorithm which overcomes all such drawbacks. We also recognize that in point-to-multipoint ATM $\mathrm{n}$ etworks, there is a possibility that the branch points will adopt different consolidation algorithms. We, therefore, investigate the interoperability issue and the impact of using different types of consolidation algorithm at the upper and lower stream branch points and show that the branch points with different consolidation algorithms can work interoperably. It is concluded from the simulation results that in order to avoid the consolidation noise and get a faster response in the network the most upper st ream branch point (the nearest one to the source) has to be implemented with a high accuracy consolidation algorithm and the lower stream branch point(s) should be implemented with a fast response consolidation algorithm. Moreover, using our proposed consolidation algorithm, fast response, low consolidation noise and low implementation complexity are achieved.
\end{abstract}

\section{Introduction}

The control of ABR service in ATM network is inherently closed loop [1. The sources send the data at the rate specified by the feedback received from the networks or destinations. There have been many rate-based congestion control schemes proposed ranging from a single bit congestion indicator [2] to explicit rate feedback to the source [3]. All of these schemes were proposed for point-topoint ABR service. In an explicit rate-based control scheme the source generates 
the Resource Management cells (RM) to the destination. We called these cells as forward RM cells (FRM). The destination used this RM cells to feedback to the source an Explicit Rate (ER) indicating an available bandwidth on the bottlenecked path connecting to it. These RM cells are called backward RM cells (BRM). The source is controlled to a minimum rate between the rate specified in BRM cell and the preset value at the source. In ATM point-to-multipoint connections, the major concern at a branch point is how to consolidate the BRM cells from destinations to the source. The branch point that wait for the BRM cells from all destinations in the multicast tree will introduce a slow response or a consolidation delay. On the other hand, if the branch point consolidates some or even a single BRM cell from the destinations and then returns to the source. This make it has a fast response but will lead to a consolidation noise because the returned BRM cell contains incomplete congestion information for some downstre am branches. Hence, the challenge of designing rate-based control algorithm in ABR point-to-multipoint communications is how to meet the different requirements requested from multiple destinations i.e. a fast response, a low consolidation noise etc. with the least expenses of an accuracy and complexity. In part of this paper, we proposed a new efficient consolidation algorithm. The algorithm offers a faster response, less consolidation noise, better link utilization and less complexity. The details of the a lgorithm will be explained in Section 3. There were several papers proposing the consolidation algorithm. The consolidation algorithm can be roughly categorized into two types the slow response with low noise and the fast response with high noise. To our best knowledge, there is no paper about interoperability issue in point-to-multipoint ABR services has been presented. We are aware that there is a possibility that the branch points will adopt different consolidation algorithm. Consequently, in this paper we investigate the inter-operability issue and the impact of of using different types of consolidation algorithm at the upper and lower stream branch points.

The organization of this paper is as follows. In Section 2, we review previously proposed related works. Our proposed consolidation algorithm is described in Section 3. Simulation results and discussions of the proposed algorithm and the interoperability issue are presented in Section 4. The paper's conclusions are drawn in the Section 5.

\section{Related Works}

There are several consolidation algorithms proposed in the previous works 44[11. A Minimum Explicit Rate (MER) register and MCI and MNI flags are widely used in the previously proposed algorithms. MER is used to temporarily store the minimum of the ER's values among those indicated by the BRM cells received from the branches and the ER calculated locally at the branch point. As mentioned earlier, we can categorize the consolidation algorithm into two types.

1. The wait-for-all consolidation algorithm. The algorithm has been proposed in [6]. In addition to MER, MCI and MNI, the algorithm requires two more counters: Number_of_BRMrecevied and Number_of_Branches. Num- 
ber_of_BRMreceived counts the number of branches from which BRM cells have been received at a branch point(after the last BRM was sent by the branch point)and Number_of_Branches stores the number of branches of a point-tomultipoint at this branch point. A branch point waits for BRM cell from all branch. When the value of this two counters get equal then the branch point returns a BRM cell with the completed congestion information to its upstream node (or source) whenever it receives a first FRM cell after completely consolidating the BRM cells. This algorithm has a slow response but does not introduce a consolidation noise.

2. The not wait-for-all consolidation algorithm. The algorithms have been proposed in [5]. The basic idea of these algorithms is that a branch point calculates ER, CI and NI value according to its local congestion level, updates MER, MCI and MNI and returns a BRM cell to its upstream node whenever it receives a FRM cell. This makes the branch point feedbacks the congestion information faster than the first type, however, because of lack of the complete congestion information the consolidation noise is quite high.

There are some proposed algorithms to achieve a fast response with low consolidation noise [9. The main idea is the branch point has to wait for all BRM cells from all branches like the algorithm proposed in 6]. If there is an overloaded condition say, the rate in current BRM cell is much less than last BRM cell, exists at the branch point. The algorithm can detect this phenomena and a branch point immediately feedbacks the BRM cell with the low ER value to the source to avoid data overflow. The branch point will send the BRM back again when BRM cell from all branches have been arrived. With this approach, we notice that the branch point seems in-active during waiting for all BRM cells. Hence, this will slow down the branch point's response. Moreover, if there is more available bandwidth, for example, from the off period of VBR traffic during this interval. This bandwidth can not be utilized by the network resulting in lower network utilization.

\section{Selective BRM Feedback Consolidation Algorithm}

In this section, an efficient consolidation algorithm is presented. We have learnt from the previously proposed papers that if the branch point does not wait for all BRM cells it will introduce the noise. On the other hand, the response is very slow if we wait for all. Based on the fact that the branch point has to send the least ER value among all BRM cells from its branches to the source. We, hence, design a consolidation algorithm called Selective BRM Feedback (SBF) that feedbacks the BRM cell to the source selectively. The branch point does not wait for all BRM cells but selects the BRM cell which contains the least ER value to send to the source. The $\mathrm{SBF}$ is designed to achieve a fast response, low consolidation noise and low complexity. It uses 3 registers at the branch point. A MER register is used for storing the ER value, a Branch_number register is used for storing the number of branch from which the branch point receives the BRM cell and a Balance_RM is used for controlling the BRM to FRM ratio to 
be 1 . The algorithm can take care of overload condition in downstream branches and can utilize, during the transient state, the available bandwidth, especially left from the VBR source in the network, without consolidation noise. It is also insensitive to the number of branches and branch point level in the network. The algorithm works as follows.

Upon the receipt of the BRM cell, the branch point checks the branch number and the ER value. If the branch number is different from the current branch number stored in Branch_number register and the received ER value is less than the current ER value stored in MER register, the MER and Branch_number are updated to the new values. Otherwise, the received BRM cell is discarded. In case BRM cell comes from the same branch number stored in the Branch_number register, the branch point always updates the ER value in MER, no matter it is more or less with respect to the previous one. Thus, these registers always preserve the latest congestion information in the downstream branches. From the above explanation, alternatively, we can show in four cases.

Case 1: if (ER from BRM $<$ MER and Branch_Number $=\mathrm{j}$ ) $\rightarrow$ Update MER and Branch_Number

Case 2: if (ER from BRM $>$ MER and Branch_Number $=\mathrm{j}) \rightarrow$ Update MER and Branch_Number

Case 3: if (ER from BRM $<$ MER and Branch_Number $\neq \mathrm{j}) \rightarrow$ Update MER and Branch_Number

Case 4: if (ER from BRM $>$ MER and Branch_Number $\neq j$ ) $\rightarrow$ Not update MER and Branch_Number

We can see that in case 1 and case 3 (ER from BRM $<$ MER) the MER is always updated. This is designed to feedback the lower value of ER from BRM quickly to the source. This technique statistically reduce the waiting time of the branch point i.e. if the branch that has the lowest ER is located nearest to the branch point, the branch point has not to wait for consolidating BRM from all branches. In case 2, where the value of ER from BRM is larger than MER and the Branch_Number is equal to $\mathrm{j}$. The branch point is also send the updated ER to source. This is our intention to let the branch point to has feature that can utilize the bandwidth left in this lowest branch. For example, if this branch share the bandwidth with the VBR traffic and in this epoch the VBR traffic is active. Hence, the traffic available for ABR service in this branch is the traffic that left from VBR. For the next epoch (normally equal to the Average Interval (AI) time in ERICA), if the VBR traffic is off. The available bandwidth for $\mathrm{ABR}$ is increased and branch point can recognize this change and feedback to the source to increase its rate. For case 4, the branch point discards the BRM cell because branch number $\mathrm{j}$ being the branch that can support the lowest rate (has the least bandwidth). The branch point will be suffered from the buffer overflow if we update the MER with the ER from BRM from the branches that has a higher rate. With this approach, the branch point is updated by the BRM cell from the most congested branch and sends it back to the source promptly without waiting for all BRM cells from all branches (that some of them might be non-responsive). The salient features of SBF are a fast response of branch point while a low consolidation noise condition is preserved. In addition, it has a low 


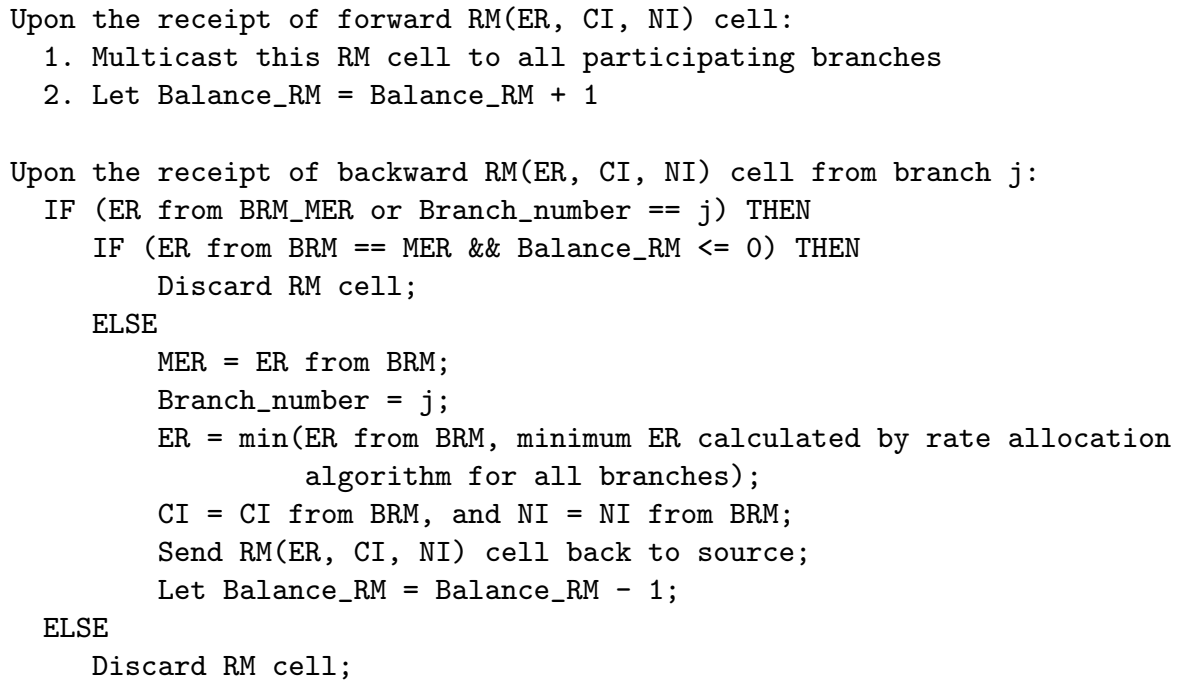

Fig. 1. Pseudo code of the proposed algorithm

implementation complexity (used only 3 registers while the previously proposed algorithms used more registers and some additional flags) The pseudo code and flow chart of the new consolidation algorithm are shown in Fig. 11 and Fig. 2 ,

\section{Simulation Results}

For convenience, all algorithms proposed in [5], [6, [9] will be referred simply to the authors'name. To refer to our propsed algorithm, we will used the term 'SBF'.

\section{Parameters Setting}

We use these values in both Network Model I and Network Model II

1. Except where indicated, all link capacity are $150 \mathrm{Mbps}$.

2. All switch-to-end system links are $50 \mathrm{~km}$ except where specified.

3. The switch target utilization is $90 \%$.

4. The switch averaging interval is set to $1 \mathrm{~ms}$.

5. The parameter Transient Buffer Exposure (TBE) is set to large values for preventing the rate decrease.

6. The source parameter Rate Increase Factor (RIF) is set to 1 .

7. Simulation time is $200 \mathrm{~ms}$, and $300 \mathrm{~ms}$ for Network Model I and Network Model II, respectively.

8. The VBR source bursts for $100 \mathrm{~ms}$ and silence for $150 \mathrm{~ms}$.

9. The Peak Cell Rate (PCR) and the Initial Cell Rate (ICR) are $150 \mathrm{Mbps}$

10. Use ERICA switch algorithm [12] as rate allocation algorithm. 


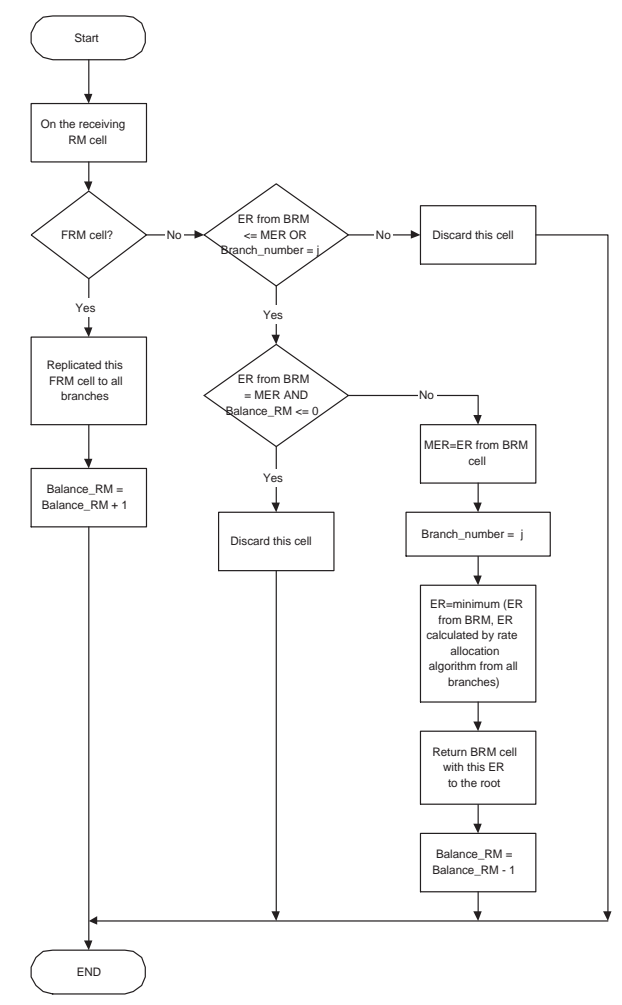

Fig. 2. Flow chart of the proposed algorithm

\subsection{Proposed Consolidation Algorithm}

In this section, we will show the simulation results of the SBF. In [9], it was shown that Fahmy is better than Roberts and Ren. Hence, we compare SBF to Fahmy only.(We actually compare SBF to Roberts and Ren. The results show that SBF has much better performance than the others in terms of response time and consolidation noise but did not show the results here because we intend to focus on the interoperability issue). The network configuration is shown in Fig. 3 Source $\mathrm{S} 1$ is a poi nt-to-multipoint connection and its destinations are $\mathrm{dS} 1, \mathrm{dS} 2$ and dS3. S4 and VBR source is a point-to-point connection and has a dS4 and dVBR destination, respectively. For convenience, we would separate the response of the network into two intervals. First interval lies between 0-80 $\mathrm{ms}$ and the second is from $80 \mathrm{~ms}$ onwards. During the first interval, the BRM cells from all destinations except for dS3 have already arrived at S1. This is because the BRM cell from dS3 has a Round Trip Time around $81 \mathrm{~ms}$. (we u se 5 microseconds per kilometer delay so the RTT for dS3 is about $81 \mathrm{~ms}$ ). In Fig. 4(a), the BRM cell from dS2 is arrived at $\mathrm{S} 1$ at $6.5 \mathrm{~ms}$. We can see that the Allowed Cell Rate (ACR) of S1 for both SBF and Fahmy is reduced from 150 Mbps to a fair share value of available bandwidth left in Link2. After this point, SBF can tell S1 to send a data according to an available bandwidth left from 


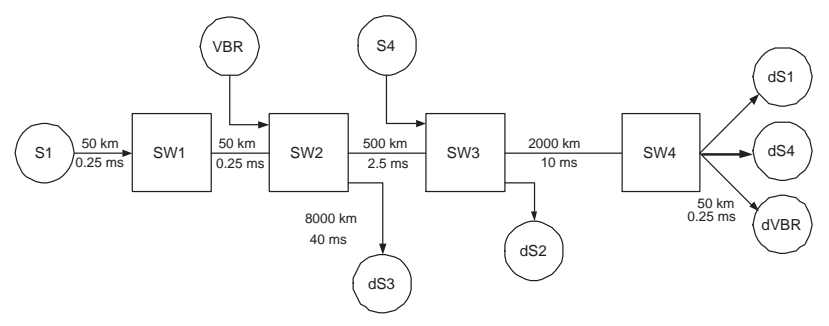

Fig. 3. Network Model I

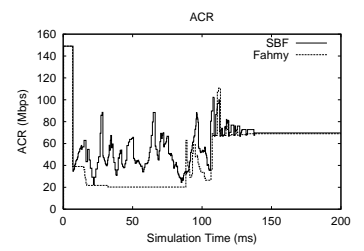

(a) Rate of S1

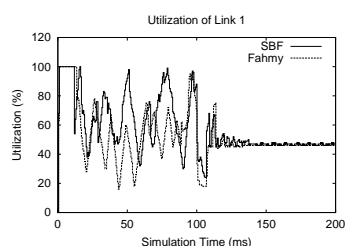

(c) Utilization of Link 1

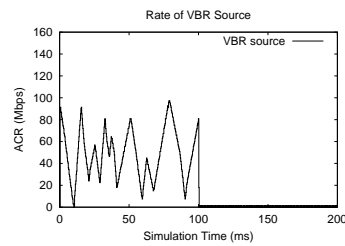

(b) VBR Rate

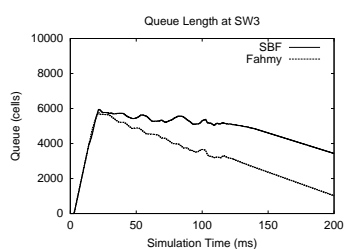

(d) Queue Length at SW3

Fig. 4. Simulation results of Network Model I

being used by the VBR source (Fig. 4(b)) while S1 of Fahmy can not. Instead, Fahmy suppresses the ACR of S1 to nearly constant low value. This leads to the lower utilization of Link1 for Fahmy comparing to SBF during the transient state (Fig. 4(c)). However, the average queue length of the SBF is a bit higher than Fahmy (Fig. 4(d)).

During the second interval, it seems no big difference between the two algorithms. The reason behind this is all BRM cells from every destinations have already reached at SW2 which mean the BRM cells from all branches are fully distributed in their path. Hence, if there is another VBR burst (after $100 \mathrm{~ms}$ ), both algorithms can detect and update the ER field in the current RM cell and send it back to S1 immediately.

\subsection{Interoperation}

In this section, We investigate the interoperation by using both the same consolidation algorithm and different consolidation algorithms at the branch points. The Network Model II is shown in Fig. 5] S1 is a source for a point-to-multipoint connection and its destinations are dS1, dS2 and dS3. The Round Trip Time (RTT) from S1 to dS1, dS2 and dS3 are $76 \mathrm{~ms}, 26 \mathrm{~ms}$ and $6 \mathrm{~ms}$, respectively. $\mathrm{S} 4$ is a source for point-to-point connection. The RTT from S4 to its destination, 


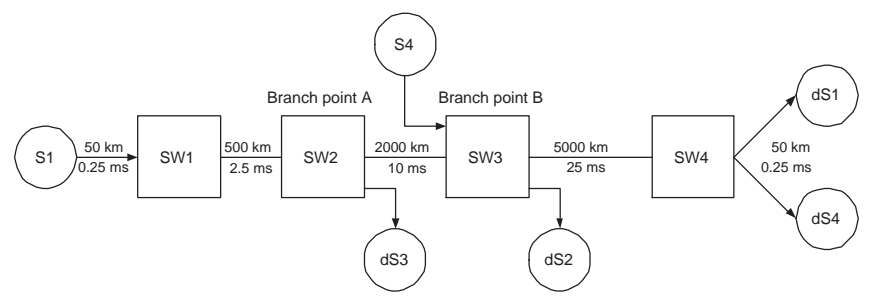

Fig. 5. Network Model II

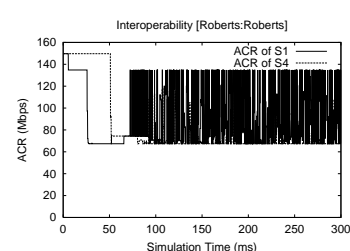

(a) Roberts

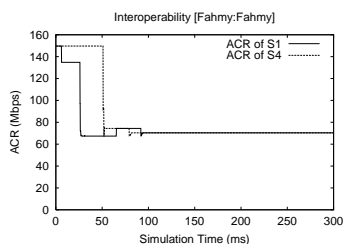

(c) Fahmy

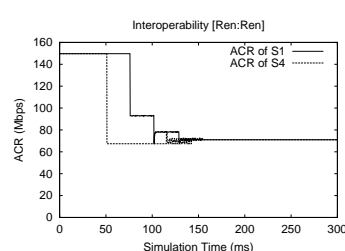

(b) Ren

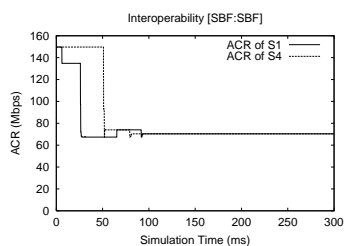

(d) SBF

Fig. 6. ACR of the source $\mathrm{S} 1$ and $\mathrm{S} 4$ using the same consolidation algorithm

dS4, is 51 ms. SW1 and SW 4 are the switching nodes and SW2 and SW3 are the branch points. The algorithms which have been described in Section 2 and Section 3 are implemented at Branch point A (SW2) and Branch point B (SW3). Branch point $\mathrm{A}$ is considered a upper stream branch point while Branch point $\mathrm{B}$ is a lower stream branch point. S1 and S4 are set to initially start sending with ICR value. This setting, on one hand, causes a high network utilization, on the other hand, exhibits a congestion and large queue length at Branch point B. To avoid the congestion, S1 and S4 should reduce their Allowed Cell Rate (ACR) to a fair share rate as fast as they can. However, the reduction of the ACR could not be occurred until receiving a turn-around RM cell from its destinations.

Fig. 6] shows the results that Branch point A and Branch point B are implemented with the same consolidation algorithm. The results of branch points that are both implemented with Roberts is shown in Fig. 6(a). Roberts is the fast response but high consolidation noise algorithm. The ACR of S1 is reduced promptly from $150 \mathrm{Mbps}$ (ICR) to $135 \mathrm{Mbps}$ (90\% of PCR as preset at the switch) when the first BRM cell from dS3 arrived at $\mathrm{S} 1$ (at $6 \mathrm{~ms}$ time). This ACR value lasts until the first BRM cell from dS2 arrived at $\mathrm{S} 1$ (at $26 \mathrm{~ms}$ time). The ACR is reduced again to $67.5 \mathrm{Mbps}$ (the fair share bandwidth between S1 and $\mathrm{S} 4$ at Branch point B). After the arrival of the farthest BRM (from dS3) at $76 \mathrm{~ms}$, S1 start to oscillate and the consolidation noise is occurred. This is 
Table 1. Simulation results for a network using the same consolidation algorithm at Branch point $\mathrm{A}$ and Branch point $\mathrm{B}$

\begin{tabular}{lllll}
\hline Branch point A & Branch point B & $\begin{array}{l}\text { Consolidation } \\
\text { algorithm } \\
\text { complexity }\end{array}$ & $\begin{array}{l}\text { Consolidation } \\
\text { noise }\end{array}$ & $\begin{array}{l}\text { Time to get to } \\
\text { fair share rate } \\
(\mathrm{ms})\end{array}$ \\
\hline Roberts & Roberts & Low & Yes & - \\
Ren & Ren & Low & No & $\geq 76$ \\
Fahmy & Fahmy & Medium & No & 26 \\
SBF & SBF & $<$ Medium & No & 26 \\
\hline
\end{tabular}

because the ACR of S1 depends on the BRM cell being sent to it by the Branch point A. If, at that moment, the BRM cell comes from dS3 then S1 is set ACR to $135 \mathrm{Mbps}$. On the other hand, if BRM cell comes from dS1 or dS2 then ACR of $\mathrm{S} 1$ will be set to $67.5 \mathrm{Mbps}$. Hence, the ACR will oscillate between $67.5 \mathrm{Mbps}$ and $135 \mathrm{Mbps}$. For S4, the ACR is dropped to a fair share value after the RTT is reach $(51 \mathrm{~ms})$. Because $\mathrm{S} 4$ is a unicast connection, it results the same graph for all figures in this section. The result of implementing Ren, which is a 'wait-for-all' BRM algorithm, at the branch points is shown in Fig. 6(b). We see that there is no consolidation noise. However, the ACR of S1 is dropped after BRM cell from dS1 (the farthest) has arrived. It takes $76 \mathrm{~ms}$ before starting to get approach a fair share rate. Comparing this slow response to Roberts' fast response time (6ms and $26 \mathrm{~ms}$ ), it is about $50 \mathrm{~ms}$ different in response time and the difference in ACR of $\mathrm{S} 1$ during this $50 \mathrm{~ms}$ period is $82.5 \mathrm{Mbps}$ (PCR minus fair share rate). This will cause an approximately $4.125 \mathrm{e} 6$ bit or 9730 excessive cells built up at Branch point B and cells may be lost if the buffer is insufficiently provided. For Fahmy and SBF, the results are shown in Fig. 6(c) and 6(d), respectively. We see that both algorithms perform identically in this network model. They exhibit a fast response and no consolidation noise, however, SBF has less in implementation complexity. Table 1 summarizes the simulation results for the network using the same consolidation algorithm at the branch points.

Fig. 7 to Fig. 10 are the simulation results of the interoperation of the branch points implementing with different consolidation algorithms. We investigate all possible combinations of the algorithms. The notation e.g. Roberts and Ren we used below each graph in all figures means Branch point A and Branch point B are implemented with Roberts and Ren, respectively. We can comprehensively analyze the results as follows;

1. Fig. 7(a), Roberts is a fast response algorithm so ACR of S1 could be dropped very fast to $135 \mathrm{Mbps}$ as explained in Fig. 6. However, Ren at Branch point B has to wait for the farthest BRM cell (from dS1) before it is able to send the BRM cell back to Branch point A. Hence, the time that S1 spends to drop its ACR to a fair share rate is $76 \mathrm{~ms}$. This slow response is equal to the longest round trip time of the network.

2. Fig. 7b) looks similar to Fig. 7(a) except for the temporary dropping to a fair share rate of ACR at $26 \mathrm{~ms}$ time. This is because Fahmy can detect an overloaded condition at Branch point B and immediately sending back a BRM 


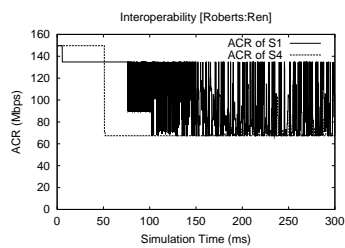

(a) Roberts and Ren

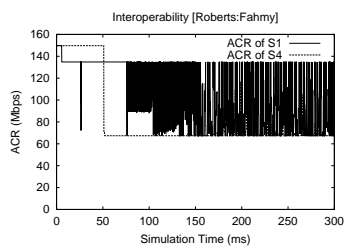

(b) Roberts and Fahmy

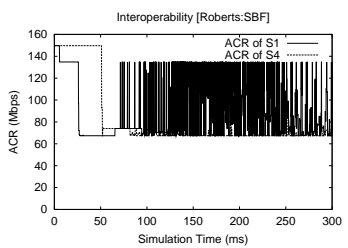

(c) Roberts and SBF

Fig. 7. ACR of the source S1 and S4 Roberts and others interoperation

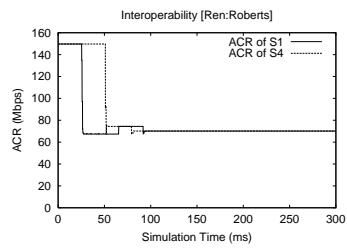

(a) Ren and Roberts

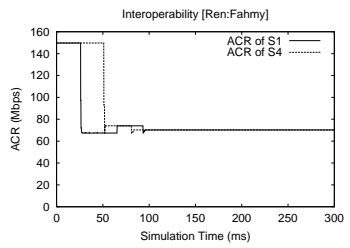

(b) Ren and Fahmy

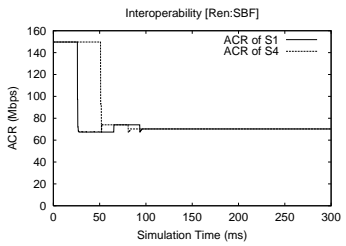

(c) Ren and SBF

Fig. 8. ACR of the source S1 and S4 Ren and others interoperation

cell with 67.5 Mbps ER value to reduce the rate of source S1. During waiting for BRM from the farthest destination, Branch point B does not send any BRM cell. Hence, the next BRM cell received by S1 come from dS3 which has 135 Mbps ER value. Then the ACR goes abruptly and will caused a lot of queue build up at Branch point B.

3. Roberts and SBF in Fig. 7(c) exhibits a similar response as inFig. 7)(b) during the $26 \mathrm{~ms}$ interval. After $26 \mathrm{~ms}$, SBF at Branch point B still feedbacks the BRM with 67.5 Mbps from a selected branch to the source and the source send the data at this rate during $26 \mathrm{~ms}$ to $76 \mathrm{~ms}$ interval. However, the ACR oscillation still exists after the $76 \mathrm{~ms}$. So far, we observe from Fig. 6 and Fig. 7 that if Branch point $\mathrm{A}$ is implemented with the algorithm that has fast response consolidation noise i.e. Robert, the ACR is oscillated no matter which algorithms are used at Branch point B.

4. Fig. 8(a) to Fig. 8(c) show the same pattern of ACR of S1 no matter what algorithm used at Branch point B. This is because Branch point $\mathrm{A}$ has to wait for all branches connecting to it before sending a BRM cell to S1. However, ACR remains at $150 \mathrm{Mbps}$ until it drops to fair share (not drop to $135 \mathrm{Mbps}$ before to fair share as in Fig. 7). Notice that in Fig. 8 ACR of S1 drops to fair share faster than that in Fig. 7(a). The reason is Roberts, Fahmy and SBF at Branch point $\mathrm{B}$ have a fast response so they can send a BRM to Branch point A within $26 \mathrm{~ms}$. while Ren has to wait for BRM from dS1.

5. The results of using Fahmy and SBF at Branch point A are shown in Fig. 9] and Fig. 10, Fahmy and SBF overcome Roberts and Ren's drawbacks. The consolidation noise exists in Fig. 7 and the slow response exists in Fig. 8 are solved. However, if Ren is used at Branch point B, S1 will take longer time than using others before getting to a fair share rate. From Fig. 8 to Fig. 10 we notice that the response of the network trends to has the same characteristics 


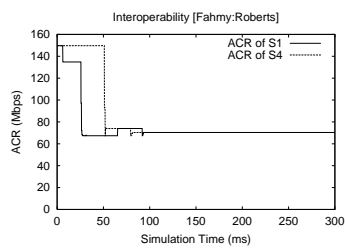

(a) Fahmy and Roberts

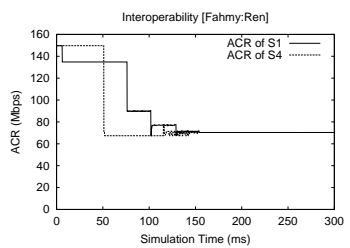

(b) Fahmy and Ren

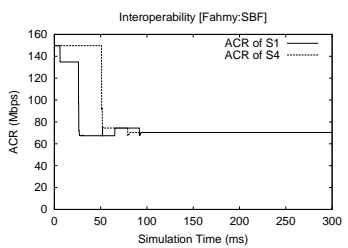

(c) Fahmy and SBF

Fig. 9. ACR of the source S1 and S4 Fahmy and others interoperation

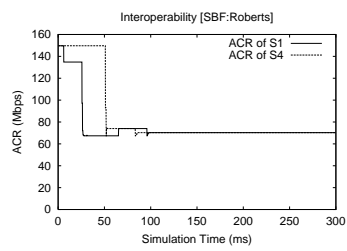

(a) SBF and Roberts

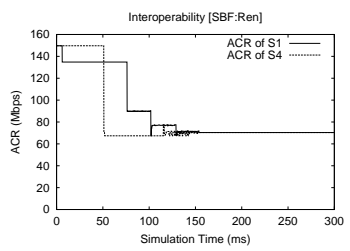

(b) SBF and Ren

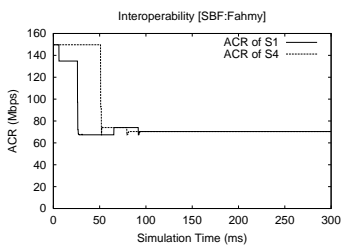

(c) SBF and Fahmy

Fig. 10. ACR of the source S1 and S4 SBF and others interoperation

as the algorithm implemented at Branch point $\mathrm{A}$. This inline with the first observation we made previously. Hence, we can conclude at this point that the most upper stream branch point dominates the response of the network while the lower stream ones have less effects. The simulation results of all cases of different consolidation algorithms used in the network are summarized in Table 2 .

\section{Conclusions}

We have proposed a new efficient consolidation algorithm. The performance of the algorithm has been evaluated using a simulation. The results show that this new algorithm meets all features required in the point-to-multipoint ABR connections. It has a faster response, better link utilization and lower implementation complexity comparing to the previously proposed one. Especially, in the network that has a VBR as a background traffic. For the interoperability issue, a variety of the previously proposed consolidation algorithms have been interoperated. We can conclude that the most upper stream branch point is the most important one in the interoperation of consolidation algorithm in point-tomultipoint network. In order to avoid the consolidation noise and get a faster response, we recommend implementing a fast response and low consolidation noise algorithm such as Fahmy or SBF at the most upper stream branch point. We found that the network performance is not much affected by the behavior of the consolidation algorithms implemented at the lower stream branch points. Hence, the implementation complexity of the algorithm should be the major issue to be considered for using in these branch points. 
Table 2. Simulation results for a network using different consolidation algorithm at Branch point $\mathrm{A}$ and Branch point $\mathrm{B}$

\begin{tabular}{lllll}
\hline Branch point A & Branch point B & $\begin{array}{l}\text { Consolidation } \\
\text { noise }\end{array}$ & $\begin{array}{l}\text { Transient } \\
\text { Response } \\
\text { time* }(\mathrm{ms})\end{array}$ & $\begin{array}{l}\text { Time to get to } \\
\text { fair share rate } \\
\text { (ms) }\end{array}$ \\
\hline \hline \multirow{3}{*}{ Roberts } & Ren & Yes & 6 & - \\
& Fahmy & Yes & 6 & - \\
& SBF & Yes & 6 & $26^{* *}$ \\
\multirow{3}{*}{ Ren } & Roberts & No & 26 & 26 \\
& Fahmy & No & 26 & 26 \\
\hline \multirow{3}{*}{ Fahmy } & SBF & No & 26 & 26 \\
& Roberts & No & 26 & 26 \\
& Ren & No & 26 & $\geq 76$ \\
SBF & SBF & No & 26 & 26 \\
& Roberts & No & 26 & 26 \\
& Ren & No & 26 & $\geq 76$ \\
\hline
\end{tabular}

* The earliest time that $S 1$ responses to the network.

** Though consolidation noise exists, $S 1$ gets to fair share rate during 26-76 ms period.

\section{References}

1. The ATM Forum: The ATM forum traffic management specification version 4.0. (1996)

2. Ramakrishnan, K.K.,Jain, R.: A Binary feedback scheme for congestion avoidance in computer networks. ACM Trans.Comp.Sys., Vol. 8. (1990) 158-181

3. Bonomi, F., Fendick, K.W.: The rate-based flow control framework for the available bit rate service. IEEE Network, (1995)

4. Tzeng, H.Y., Siu, K.Y.: On Max-min fair congestion control for multicast ABR service in ATM. IEEE Journal on Selected Areas in Communications, (1997)

5. Roberts, L.: Rate based algorithm for point to multipoint ABR service. ATM Forum 94-0772R1, (1994)

6. Ren, W., Siu, K.Y., Suzuki, H.: On the performance of congestion control algorithms for multicast ABR service in ATM. IEEE ATM'96 Workshop, (1996)

7. Cho, Y-Z., Lee, S-M., Lee, M-Y.: An Efficient Rate Algorithm for Point-toMultipoint ABR Service. IEEE GLOBECOM, (1997)

8. Fahmy, S., Jain, R., Kalyanaraman, S., Goyal, R., Vandalore, B., Kota, S., Samudra, P.: Design and Evaluation of Feedback consolidation algorithm for ABR pointto-multipoint Connections in ATM Networks. IEEE INFOCOM'98, (1998)

9. Fahmy, S., Jain, R., Kalyanaraman, S., Goyal, R., Vandalore, B., Kota, S., Samudra, P.: Feedback consolidation algorithm for ABR point-to-multipoint connections. ATM Forum 97-0615, (1997)

10. Jiang, T., Zegura, E.W., Ammar, M.: Improved consolidation algorithms for pointto-multipoint ABR service. IEEE, 0-7803-4874-5/98. (1998)

11. Rangsinoppamas, N., Jaruvitayakovit, T., Sa-Niamsak, W., Prapinmongkolkarn, P.: Compromised Consolidation Algorithm for Point-to-Multipoint ABR Service in ATM Networks. ATCS'99, (1999)

12. Jain, R., Kalyanaraman, S., Goyal, R., Fahmy, S., Viswanathan, R.: ERICA switch algorithm: A complete description. ATM Forum 96-1172, (1996) 